\title{
Quantum cascade laser infrared spectroscopy of single cancer cells
}

\author{
Imran I. Patel, ${ }^{1}$ Vijayakumar P. Rajamanickam, ${ }^{1}$ Andrea Bertoncini, ${ }^{1}$ Francesca Pagliari, ${ }^{1}$ Luca \\ Tirinato, ${ }^{1}$ Sergey Laptenok, ${ }^{1}$ Carlo Liberale ${ }^{1}$ \\ ${ }^{1}$ BESE Division, King Abdullah University of Science and Technology (KAUST), Thuwal, Makkah, Kingdom of Saudi Arabia \\ carlo.liberale@kaust.edu.sa
}

\begin{abstract}
Quantum cascade laser infrared spectroscopy is a next generation novel imaging technique allowing high throughput and high resolution label-free imaging of cells. We show after spectral pre-processing, identification of different cancer cell populations within minutes. OCIS codes: $110.0110,300.0300,170.0170$.
\end{abstract}

Infrared (IR) spectroscopy exploits the ability of cellular biomolecules to absorb in the mid-IR region $(\lambda=2.5-25$ $\mu \mathrm{m})$ through vibrational transitions of chemical bonds. A typical instrument consists of a mid IR source, a Michelson interferometer and a liquid nitrogen cooled mercury cadmium telluride detector [1]. Application of a new benchtop Infrared micro-spectrometer for high throughput imaging with high resolution has been now been utilised for label-free microscopy in cell biology [2]. The imaging system is capable of achieving high spectral brightness, which was previously only attainable at a synchrotron facility. The system contains multiple tunable quantum cascade lasers (QCL) combined by an optical multiplexer which are directed towards the sample, ultimately the absorbance is detected by a microbolometer camera.

The application of infrared wavelengths within the same region of that of a typical cell size $(5-20 \mu \mathrm{m})$ have been demonstrated to cause significant scattering during single cell analyses, distorting the cell spectrum and causing a loss of absorbance intensities within the region of cell size and organelles. Many baseline correction techniques correct for optical aberrations, while Bassan et al, have provided an algorithm for which the dispersive effects can be corrected by using a pure reference spectrum and an iterative process which improves the dataset in relation to the reference spectrum [3].

In this paper, we image MCF-7 cell populations incubated under low and high glucose medium using novel QCL spectral imaging. The effect of optical aberrations are minimized from spectra using differing baseline correction techniques, whilst multivariate analysis using PCA allows cell populations to be characterized.

MCF-7 cells were grown in either low $(1 \mathrm{~g} / \mathrm{L})$ or high glucose $(4.5 \mathrm{~g} / \mathrm{L})$ medium and incubated in a humidified atmosphere with $5 \% \mathrm{CO}_{2}$ at $37^{\circ} \mathrm{C}$. Cell suspensions were then spotted at $(50,000$ cells / $\mu$ l) for single cell layers onto Low E-reflective glass slides and dried. Infrared spectra were acquired using a Spero ${ }^{\mathrm{TM}} \mathrm{QCL}$ microscope (Daylight solutions). Cells were analysed using a X12.5 high magnification ( $650 \mu \mathrm{m}$ X $650 \mu \mathrm{m}$ field of view) onto a $480 \times 480$ pixel focal plane array (1.36 $\mu \mathrm{m}$ pixel size).

Raw spectra were baseline corrected using second order differentiation and vector normalization. Multivariate analysis was carried out using MATLAB in-house written scripts (IRootLab) [4-6]. Exploratory principal component analysis (PCA) was primarily performed as an unsupervised technique producing a scores and loadings plot from derived PCs of the mean-centered, processed spectra. 
To determine whether QCL based infrared spectroscopy is able to distinguish MCF-7 cells fed with high and low glucose medium, QCL spectral images were acquired from cells in transflection mode on Low-E reflective slides. After spectral acquisition we can see from comparison of the average baseline-uncorrected spectra that the peaks are similar but those from low glucose (blue) require baseline correction (Fig 1A). Subsequently, 120 spectra were taken from images of MCF-7 cells and applied to pre-processing using second order differentiation and multivariate analysis using PCA analysis to determine unsupervised segregation (Fig. 1B). MCF-7 cells showed the greatest segregation across PC1 with no areas of overlap. We can clearly see that the two classes are inherently clustered with both high glucose (red) and low glucose (blue) from each media clearly segregated. From the PCA loadings plot, the wavenumbers responsible for segregation of the high glucose and low glucose cells are $1680 \mathrm{~cm}^{-1}$ (Amide I), $1550 \mathrm{~cm}^{-1}$ (Amide II) $1080 \mathrm{~cm}^{-1}$ (DNA) and $1740 \mathrm{~cm}^{-1}$ (lipid) (Fig. 1C).

A

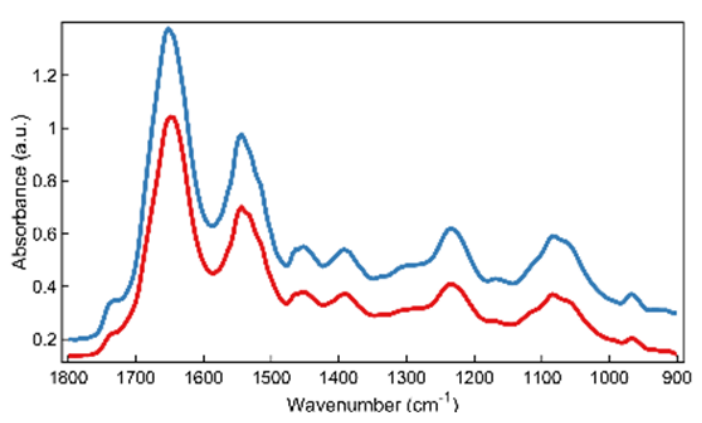

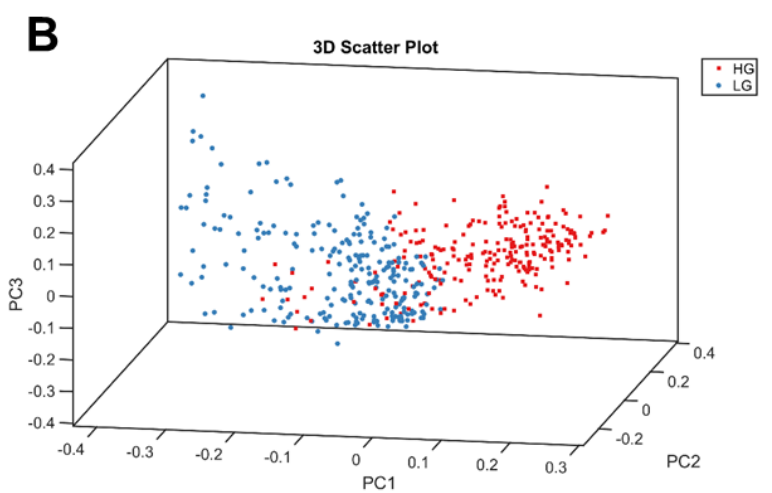

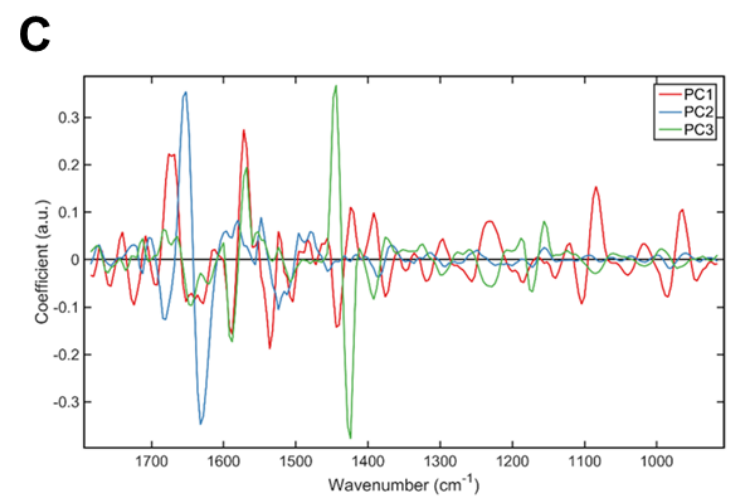

Figure 1) Uncorrected average spectra of high (red) and low (blue) glucose (A). 3D PCA scores plot of high glucose (red) vs those of low glucose (blue) spectra (B). PCA loadings plot identifying the bio-molecular changes across PC1 (red), PC2 (blue) and PC3 (green).

In conclusion, QCL infrared imaging of cells with appropriate pre-processing and multivariate analysis can distinguish cell populations within minutes. 


\section{References}

1. M. J. Baker, J. Trevisan, P. Bassan, R. Bhargava, H. J. Butler, K. M. Dorling, P. R. Fielden, S. W. Fogarty, N. J. Fullwood, K. A. Heys, C. Hughes, P. Lasch, P. L. Martin-Hirsch, B. Obinaju, G. D. Sockalingum, J. Sule-Suso, R. J. Strong, M. J. Walsh, B. R. Wood, P. Gardner, and F. L. Martin, Nature Protocols 9, 1771-1791 (2014).

2. K. Yeh, S. Kenkel, J. N. Liu, and R. Bhargava, Anal Chem 87, 485-493 (2015).

3. P. Bassan, H. J. Byrne, F. Bonnier, J. Lee, P. Dumas, and P. Gardner, Analyst 134, 1586-1593 (2009).

4. F. L. Martin, J. G. Kelly, V. Llabjani, P. L. Martin-Hirsch, Patel, II, J. Trevisan, N. J. Fullwood, and M. J. Walsh, Nat Protoc 5, 1748-1760 (2010).

5. J. Trevisan, P. P. Angelov, A. D. Scott, P. L. Carmichael, and F. L. Martin, Bioinformatics 29, 1095-1097 (2013).

6. Patel, II, J. Trevisan, P. B. Singh, C. M. Nicholson, R. K. Krishnan, S. S. Matanhelia, and F. L. Martin, Anal Bioanal Chem 401, 969-982 (2011). 